\title{
Choice Overload, Satisficing Behavior, and Price Distribution in a Time Allocation Model
}

\author{
Francisco Álvarez, ${ }^{1}$ José-Manuel Rey, ${ }^{2}$ and Raúl G. Sanchis ${ }^{3,4}$ \\ ${ }^{1}$ Department of Quantitative Economics, Complutense University of Madrid, Campus de Somosaguas, 28223 Madrid, Spain \\ ${ }^{2}$ Department of Economic Analysis, Complutense University of Madrid, Campus de Somosaguas, 28223 Madrid, Spain \\ ${ }^{3}$ RCC at Harvard University, 26 Trowbridge Street, Cambridge, MA 02138, USA \\ ${ }^{4}$ Department of Economics, Carlos III University of Madrid, c/Madrid 126, 28903 Madrid, Spain \\ Correspondence should be addressed to José-Manuel Rey; j-man@ccee.ucm.es
}

Received 1 November 2013; Revised 6 February 2014; Accepted 7 February 2014; Published 20 March 2014

Academic Editor: Juan C. Cortes

Copyright ( 2014 Francisco Álvarez et al. This is an open access article distributed under the Creative Commons Attribution License, which permits unrestricted use, distribution, and reproduction in any medium, provided the original work is properly cited.

\begin{abstract}
Recent psychological research indicates that consumers that search exhaustively for the best option of a market product-known as maximizers-eventually feel worse than consumers who just look for something good enough - called satisficers. We formulate a time allocation model to explore the relationship between different distributions of prices of the product and the satisficing behavior and the related welfare of the consumer. We show numerically that, as the number of options becomes large, the maximizing behavior produces less and less welfare and eventually leads to choice paralysis-these are effects of choice overload-whereas satisficing conducts entail higher levels of satisfaction and do not end up in paralysis. For different price distributions, we provide consistent evidence that maximizers are better off for a low number of options, whereas satisficers are better off for a sufficiently large number of options. We also show how the optimal satisficing behavior is affected when the underlying price distribution varies. We provide evidence that the mean and the dispersion of a symmetric distribution of prices-but not the shape of the distributioncondition the satisficing behavior of consumers. We also show that this need not be the case for asymmetric distributions.
\end{abstract}

\section{Introduction}

Facing a consumer decision in the market in the Western world today entails considering a very large number of options. From shopping at a modern supermarket to buying online, daily experience seems to highlight that a consumer choice is quite a demanding task in terms of processing all the available information in the relevant market.

Psychological research $[1,2]$ has recently revealed several adverse effects associated with such overload of choices, mainly the so-called paradox of choice and the paralysis effect. The former suggests the fact that an explosion of choice affects consumers' welfare in a way that "more is less." This is manifested in the shape of an inverted $U$ for the welfare with respect to number of options, what implies that a consumer is less satisfied when facing a choice problem with a large number of options. The paralysis effect refers to situations in which the overload is perceived so important that consumers choose not to choose. Rational time allocation was proposed in [3] as an underlying framework to account for choice overload effects in a market with an increasing number of options. While choice overload is not considered a universal phenomenon [4], the analysis of the model in [3] reproduced the effects described above under certain natural conditions, for example, about consumer's preferences over time uses.

Further research from both economics and psychology $[5,6]$ suggests the following provoking idea: consumers who exhaustively search for the best product option in the market-maximizers-eventually feel worse than consumers who just look for something "good enough" - called satisficers, despite the fact that maximizers get a better deal. To analyze the "satisficing claim" above a more precise definition of "satisficer" is required, which seems to be lacking in the literature. We propose in this paper a definition for satisficer that fits well within the time allocation model in [3].

We consider a typical profile of consumer, that is, with a balanced preference among different uses of time and with a linear search cost (see Section 2 and [3] for more 
details). Then, given an underlying probability distribution for the prices of the product, the welfare issue is analyzed by scanning different degrees of satisficer behavior for an increasing sequence of the number of product options/prices.

The basic inputs of the model thus are the price distribution and a (growing) sequence of product options, and the main output is a classification of satisficing behaviors in terms of welfare for each number of options. In particular, the analysis yields a whole of satisficer conducts-varying with the number of given product options-that are better off than the corresponding maximizer. Different price distributions are considered and the satisficing responses are analyzed. Similar qualitative results are obtained for different distributions that confirm the "satisficing claim." According to our analysis, satisficers tend to be better off than maximizers beyond certain number of options, whereas maximizers are better off for a relatively small number of options. The sensitivity of the results with respect to statistical parameters of the distribution (mean and dispersion of prices) as well as with respect to the shape of distribution itself is also addressed. A significant finding here is that, for symmetric distributions, changes in the shape of the distribution that preserve mean and dispersion do not influence the satisficing behavior. It is also shown that for asymmetric distributions that need not be the case.

The main features of the model are introduced in Section 2, the results obtained from the model analysis and their discussion are presented in Section 3, and some conclusions are gathered in Section 4.

\section{Time Allocation, Price Distribution, and Satisficing Behavior}

Our approach to the problem of choice overload and the "satisficing claim" relies on a version of the model of rational time allocation among competing uses of time that was introduced in [3]. A consumer decides how to spend her total available time $(T)$ in three different rival uses of time; so that

$$
T_{s}+T_{f}+T_{w}=T, \quad T_{s}, T_{f}, T_{w} \geq 0,
$$

where $T_{s}$ is shopping time, $T_{w}$ is working time, and $T_{f}$ is nonworking time, or free time.

The consumer typically faces a large number of given market options for every product. We focus on her decision about acquiring a single product among the many versions of the product offered in the market. Let $N$ be the total number of product options that are available and that she could inspect to make her buying decision. Let $0 \leq a \leq 1$ denote the fraction of the total number $N$ that a consumer may decide to check, so that $n=a N$ is the number of options that she actually looks over. We may think of $a$ as the strategy adopted ex-ante by the consumer to solve her shopping problem, so that $a=1$ corresponds to a maximizer-she explores all possible market options-whereas $0<a<1$ refers to an $a$-satisficer-the one that looks only at a fraction $a$ of the options before making her buying decision.

Consumer's total expenditure is bounded from below by some quantity $G$ which is a function of the number of checked options $n, G:=G(n)$. The consumer's decision is subject to the budget constraint

$$
G(n) \leq w T_{w}+V,
$$

where $w$ is the wage rate per unit of working time $\left(T_{w}\right)$ and $V$ is nonworking income or savings. Since $G(n)$ represents the best deal when $n$ options have actually been considered, it typically depends on $n$ in a nonincreasing fashion.

We assume that a set of $N$ prices of the different market options has first been obtained as a random sample of length $N$ of an underlying distribution $\mathscr{F}$, so that the consumer has to make her decision about checking a subsample of length $0 \leq n \leq N$ of the $N$ market options. The cases of $\mathscr{F}$ normal and uniform will be first considered below.

We also adopt the point of view that a representative consumer searching for the best deal in fact faces $G(n)=$ $\mathbf{E}\left[\min \left\{p_{1}, p_{2}, \ldots, p_{n}\right\}\right]$, that is, she solves her decision problem with $G(n)$ defined as the expected minimum price from a subsample of length $n$ of the available prices.

Since the expected best price decreases as the number of seen options increases, there is incentive to look for more options and consequently to spend more time searching in the market. Nevertheless, searching for more options is time consuming. Let $\tau=\tau(n)$ denote the minimum time that is required to find and evaluate $n$ options of the desired product. The consumer thus faces the time constraint

$$
T_{s} \geq \tau(n) .
$$

Notice that the shopping time floor defined by $\tau(n)$ may depend on the search efficiency of the consumer and also on the organization of the market of the product. In general, it may be assumed that $\tau(n)$ is a nondecreasing function of $n$. In this paper, we assume a linear form for the cost of inspection time, that is, $\tau(n)=c n$, so that $c>0$ is the inspection time per unit.

Under the standard assumption of rational behavior, the consumer seeks to maximize her welfare. Since this depends on the way she decides to use her time, her welfare can be written as a function

$$
U\left(T_{s}, T_{f}, T_{w}\right) .
$$

Therefore, the consumer choice problem is an optimization problem in which she determines the time distribution $\left(T_{s}, T_{f}, T_{w}\right)$ that maximizes her welfare (4) subject to the constraints (1), (2), and (3). Given the number $N$ of market prices, the optimal search strategy $n^{*}$ for the satisficer's problem-along with suboptimal strategies that beat the maximizer's welfare status-is obtained as a by-product of the time allocation solution.

\section{Results and Discussion}

We consider a standard profile of consumer; that is, she has balanced preferences among different uses of time in addition to linear inspection cost (see case \#1 in [3], where other profiles are considered). To illustrate the solution output, the model is first solved numerically for two natural 


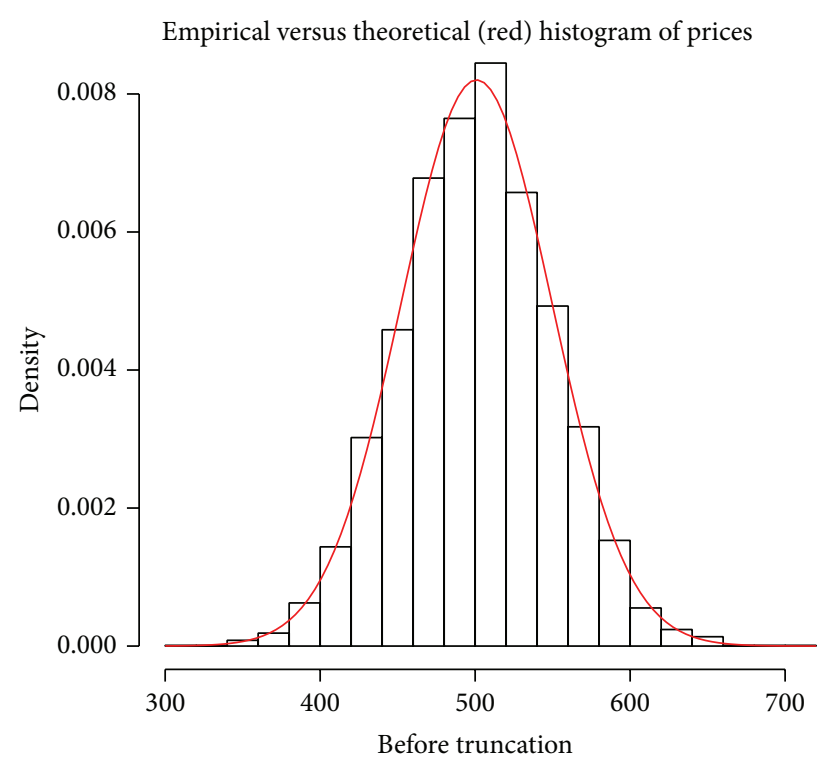

(a) Histogram of prices

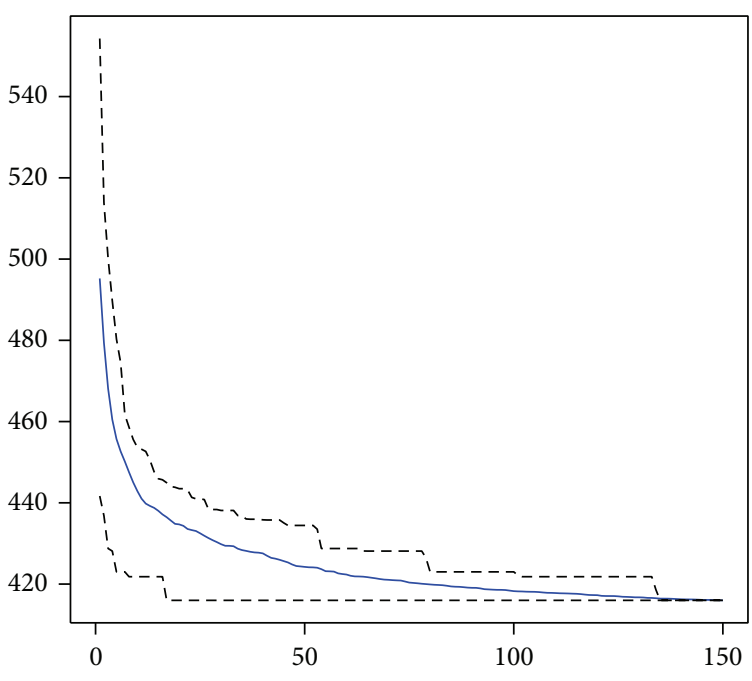

(b) Expected minimum price as $N$ increases-the error estimation intervals correspond to the $10 \%$ and $90 \%$ percentiles of the empirical sample distributions

FIgURE 1: Price distribution and expected minimum prices, case of normal distribution $N(500,50)$.

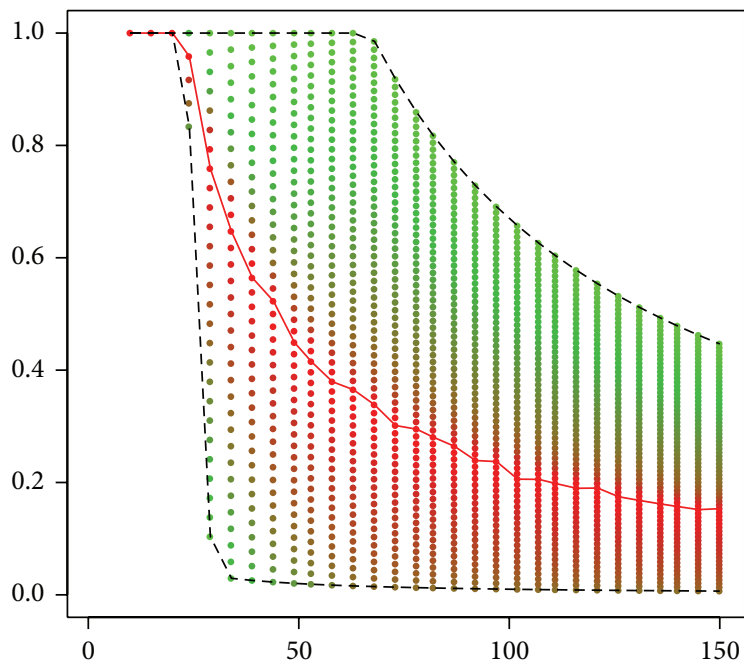

(a) Satisficer correspondence (a-range better than maximizer for each $N$ ) and best satisficer curve (in red)

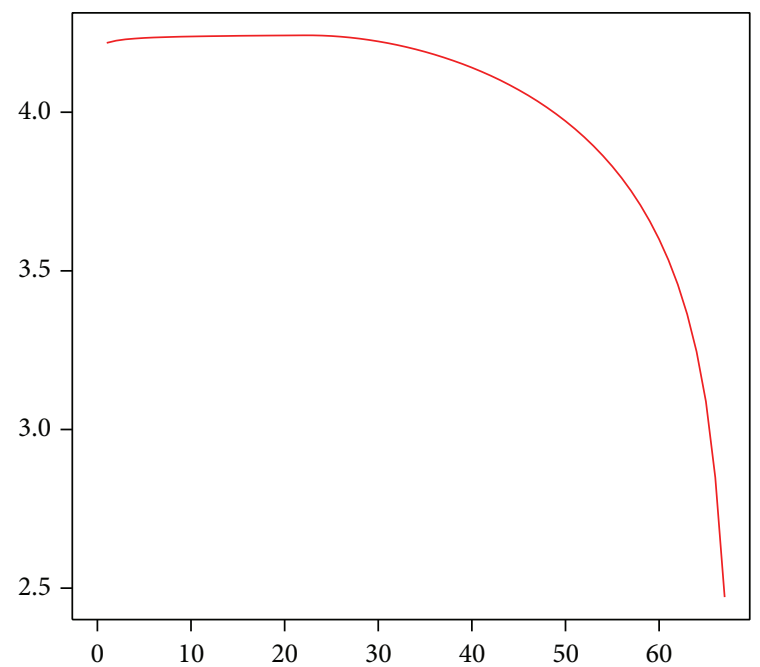

(b) Utility as $n$ increases $(N=150)$

FIGURE 2: Satisficing behavior and welfare, case of normal distribution $N(500,50)$.

price distributions, namely, normal and uniform. Table 1 summarizes the essential functional forms and parameter values for the reference case that will remain fixed for the sequel.

We first consider the case that prices are normally distributed. Synthetic price data are displayed in Figure 1(a) corresponding to $N=150$ along with the theoretical density, whereas the estimated expected minimum price as a function of $N$ is shown in Figure 1(b).

Figures 2(a) and 2(b) show the output of the numerical analysis of the model. Optimal welfare as a function of $N$ can be seen in Figure 2(b): it is apparent that welfare exhibits a shape of inverted- $U$, which indicates that the consumer suffers from choice overload: although her welfare increases when the number of product options is low, it starts to decrease when the market size $N$ reaches certain value $N^{*}$ (in this case, $N^{*}=22$ ). The welfare analysis for different satisficer strategies is represented in Figure 2(a). The picture represents the graph of the correspondence that, for each $N$, gives the range of $a$-satisficers (looking over a sample of $a N$ options out of the total $N$ ) that are better off than a maximizer (looking over the $N$ options). Specifically, let $U^{*}(n)$ be 


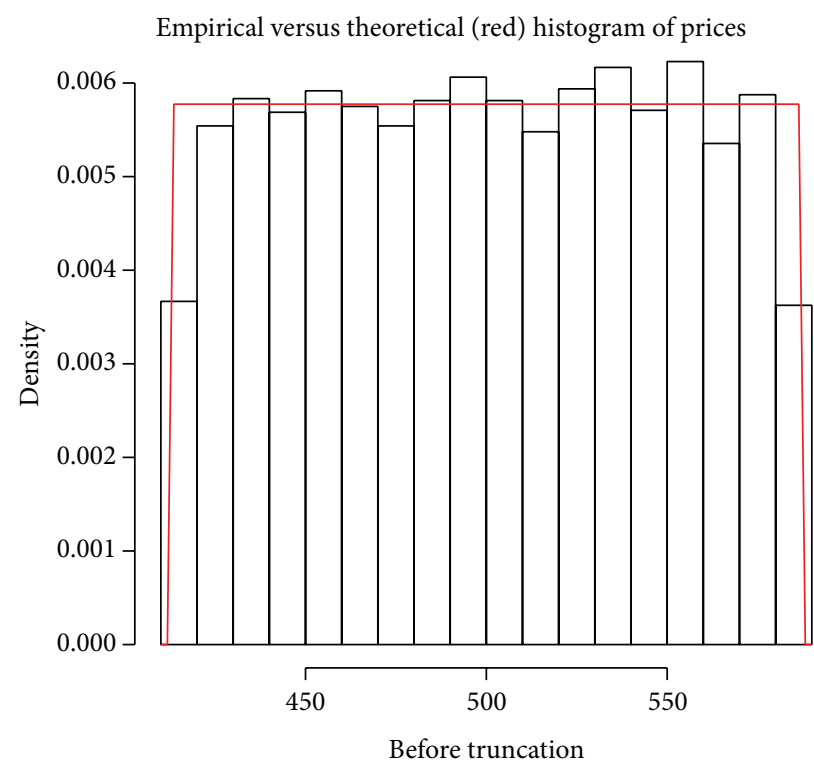

(a) Histogram of prices

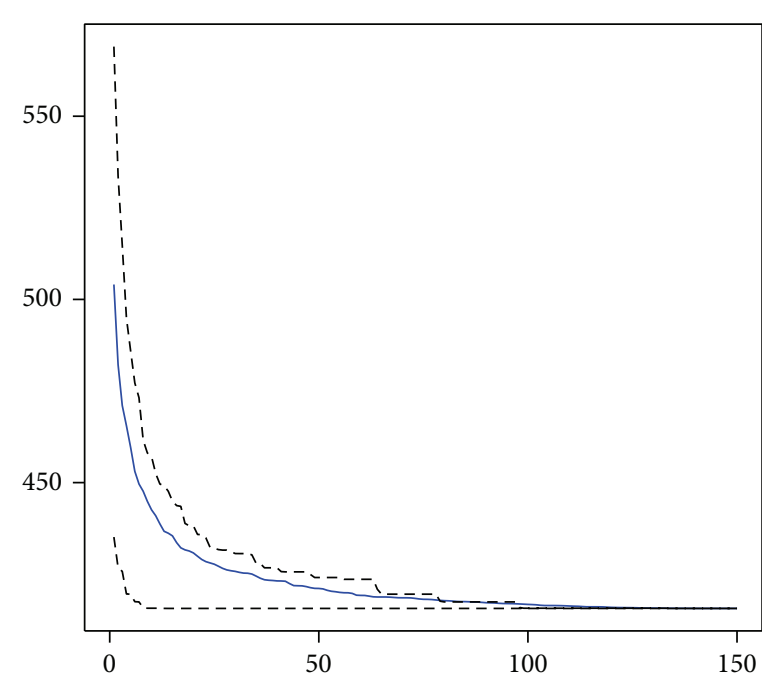

(b) Expected minimum price as $N$ increases-the error band corresponds to the $10 \%$ and $90 \%$ percentiles of the empirical sample distributions

FIGURE 3: Price distribution and expected minimum prices, case of uniform distribution with mean $\mu=500$ and standard distribution $\sigma=50$.

TABLE 1: Essential parameter values.

\begin{tabular}{lcc}
\hline Concept & Function & Parameter values \\
\hline Total time & See $(1)$ & $T=200$ \\
Budget & See $(2)$ & $(V, w)=(0,6.5)$ \\
Utility & $U=a_{1} \ln T_{s}+$ & $\left(a_{1}, a_{2}, a_{3}\right)=$ \\
Search & $a_{2} \ln T_{f}+a_{3} \ln T_{w}$ & $(0.25,0.5,0.25)$ \\
cost & $\tau(n)=c n$ & $c=2$ \\
Market & & $10 \leq N \leq 150$ \\
options & & \\
\hline
\end{tabular}

the optimal welfare of the consumer problem as a function of the number $n$ of checked options. Then Figure 2(a) shows the graph of the satisficer correspondence, defined by

$$
\begin{aligned}
A(N)=\left\{a \in[0,1]: U^{*}(a N)\right. & \left.\geq U^{*}(N)\right\}, \\
10 & \leq N \leq 150 .
\end{aligned}
$$

The red curve $a^{*}=a^{*}(N)$ inside the shaded region corresponds to the best satisficer, which enjoys maximal welfare for each value of $N$. The fading from red to green in the graph of $A(N)$ corresponds with the decrease in welfare as $a$ moves away from the red curve $a^{*}$. When $N$ is so large that the feasible set of time uses is empty, say, for $N>N_{p}$, there is not a solution for the consumer problem, what may be interpreted as choice paralysis, as it was explained in [3]. This effect may be noticed in the blank region on the upper right corner of Figure 2(a): from $N=N_{p}=67$ on, it is no longer feasible to explore more options and thus the upper contour of the graph of the correspondence decays as $N_{p} / N$.

Figures 3(a)-3(b) and 4(a)-4(b) show the numerical input and output of the model in the case of a uniform price distribution with the same mean $(\mu=50)$ and standard deviation $(\sigma=50)$ as the normal distribution considered above.

From the numerical analysis above it is apparent that the model output does not differ qualitatively whether prices are distributed normally or uniformly. The graphs in Figures 2 (a) and 4(a) show that a maximizing strategy gives maximal welfare for low values of $N$, whereas a whole variety of satisficing strategies produces more welfare than the maximizing strategy for larger values of $N$, due to choice overload.

Under choice overload effects, the typical graph of the satisficer correspondence $A(N)$ is as shown in Figure 5. Specifically, for values of $N$ below $N^{*}$, the graph of the satisficer correspondence is in fact the curve corresponding to $a=1$-the maximizer's curve-because checking all options is the best strategy. This defines the maximizer zone in Figure 5. Then choice overload enters the scene and maximizing is no longer the best strategy in terms of welfare. Rather, for each $N$ beyond $N^{*}$, there is an interval of $a$-satisficers that are better off than maximizers. Within the satisficer zone I in Figure 5 the size of the satisficer intervals and the height of the graph increase for $N$ between $N^{*}$ and $N_{p}$. Beyond the number $N_{p}$ of options, the maximizer experiences paralysis-no time allocation is feasibleand the fraction of options that can be checked decreases as $N$ increases. In this region-the satisficer zone II in Figure 5-the width of the correspondence graph decreases as the number of options increases.

Notice that Figures 2(a) and 4(a) look strikingly similar. In fact, the threshold numbers between the three regions of the graph are $N^{*}=22$ and $N_{p}=67$ in both cases, which is remarkable. The resemblance between the two satisficer correspondences suggests that satisficing behaviors may not respond to the way in which prices are distributed, as long 


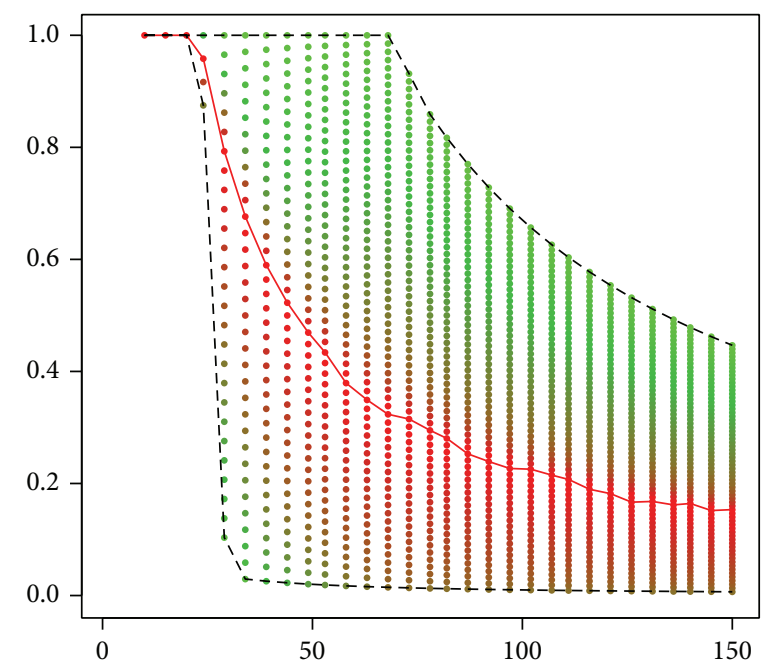

(a) Satisficer correspondence ( $a$-range better than maximizer for each $N$ ) and best satisficer curve (in red)

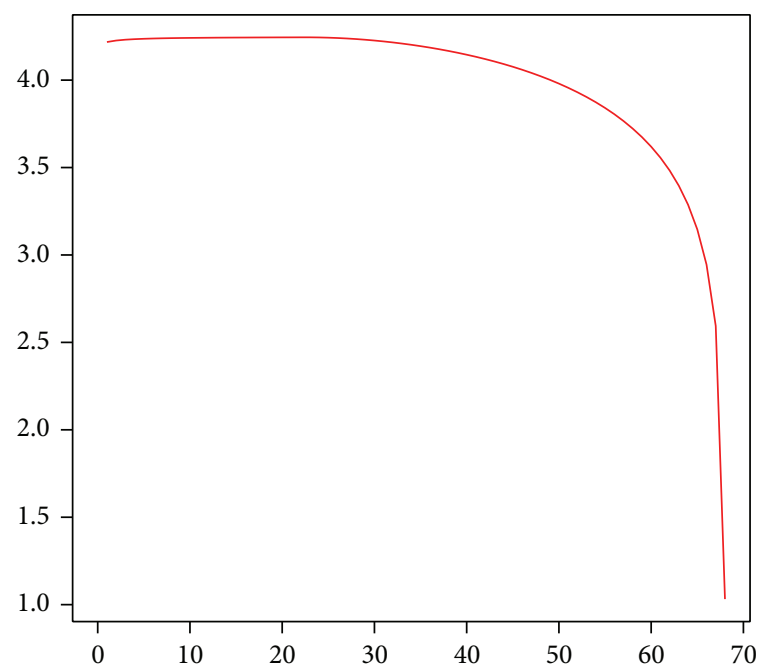

(b) Utility as $n$ increases $(N=150)$

FIGURE 4: Satisficing behavior and welfare, case of uniform distribution with $\mu=500$ and $\sigma=50$.

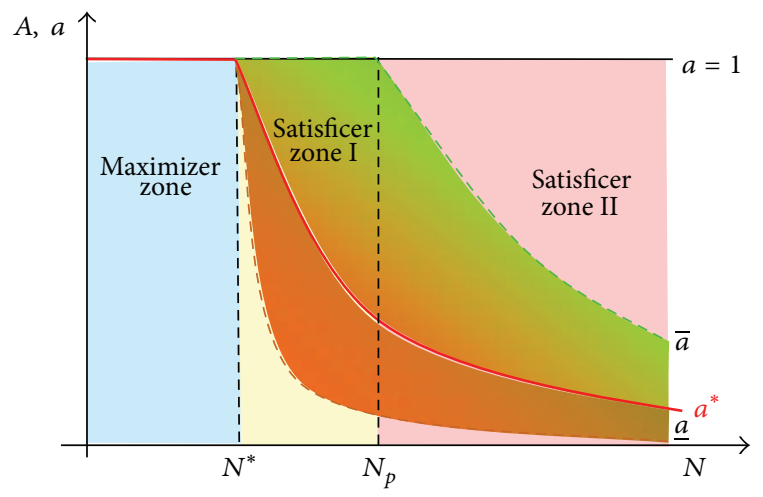

FIGURE 5: Model output: typical satisficer correspondence $A(N)$ and best satisficer curve $a^{*}(N)$. The two curves $\bar{a}$ and $a$ limit the contour of the graph of $A(N)$. They define three different satisficer regions. In the maximizer zone where $a(N)=\bar{a}(N)$, welfare is optimal when all options are checked and the best satisficer is the maximizer. In the satisficer zone I, the consumer is able to choose as a maximizer but optimal welfare is obtained looking over a fraction $a^{*}$ of $N$ which is decreasing as $N$ increases. In the satisficer zone II, the optimal fraction is also decreasing but the consumer cannot act as a maximizer, who is experiencing choice paralysis.

TABLE 2: Shocks $a_{h}$ below are independent and uniformly distributed in $[-\theta, \theta]$ where $\theta$ is adjusted as $H$ changes so as to keep $\sigma$ constant.

\begin{tabular}{lcccc}
\hline & $\mu$ & $\sigma$ & $\mathscr{F}$ & $H$ \\
\hline Green line & & & & $H=2$ \\
& 700 & \multirow{2}{*}{150} & $\mu+\frac{1}{H} \sum_{h} a_{h}$ & $H=20$ \\
Red line & & & & $H=30$ \\
\hline
\end{tabular}

as they have a common mean and standard deviation, as in the two cases studied above. To further explore this claim, we depart from a normal distribution $N(\mu=700, \sigma=150)$ as the benchmark case and consider a family of distributions $\mathscr{F}_{H}$ obtained as an average of $H$ identical shocks around $\mu$, which are independent and uniformly distributed. This allows us to rank $\mathscr{F}_{H}$ from the uniform distribution (when $H=1$ ) up to an asymptotically normal distribution (when $H$ is very large). Specifically, we consider that each price observation $p$ is obtained as

$$
p=\mu+\frac{1}{H} \sum_{h} a_{h},
$$

where $\left\{a_{1}, \ldots, a_{H}\right\}$ are the iid shocks uniformly distributed on some interval $[-\theta, \theta]$. Notice that $\mu$ is the mean of $\mathscr{F}_{H}$ regardless of the number of shocks. Additionally, $\theta$ is changed as $H$ varies so as to keep the variance of $\mathscr{F}_{H}$ at its benchmark value. It is clear that $\mathscr{F}_{H}$ remains symmetric for any $H$. Roughly, the distance to normality is parameterized by the number of shocks. We consider three distributions generated by intermediate values of $H$ between the uniform and the normal cases (see Table 2). The output of the model for this set of distributions is displayed in Figures 6(a) and 6(b). The analysis produces best satisficer curves $a^{*}(N)$ and satisficer correspondences $A(N)$ that are all practically indistinguishable. Also, no significant difference is perceived in the related best satisficer's utilities, as it can be seen in Figure 6(b). This numerical exercise supports our claim that different symmetrical distributions with common mean and dispersion have the same influence either in the satisficer behavior or her optimal welfare.

Observe that, once the essential parameters of the model have been fixed, the satisficer correspondence $A(N)$ and the best satisficer curve $a^{*}(N)$ depend only on the price distribution $\mathscr{F}$. The numerical experiment above strongly suggests that if $\mathscr{F}_{1}$ and $\mathscr{F}_{2}$ are different symmetric distributions with the same means $\mu_{1}=\mu_{2}$ and standard deviations $\sigma_{1}=\sigma_{2}$ 


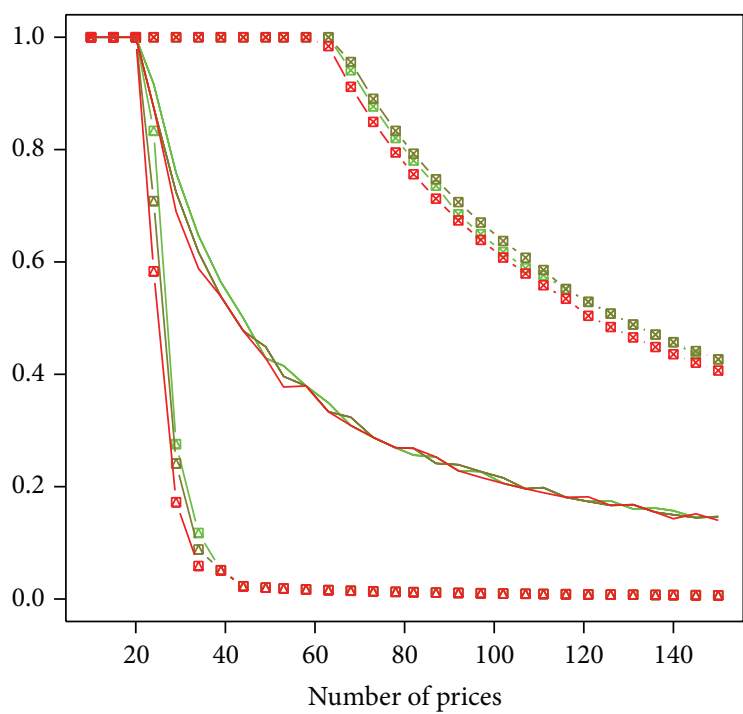

(a) Satisficer correspondences $A(N)$ and best satisficer curves $a^{*}(N)$

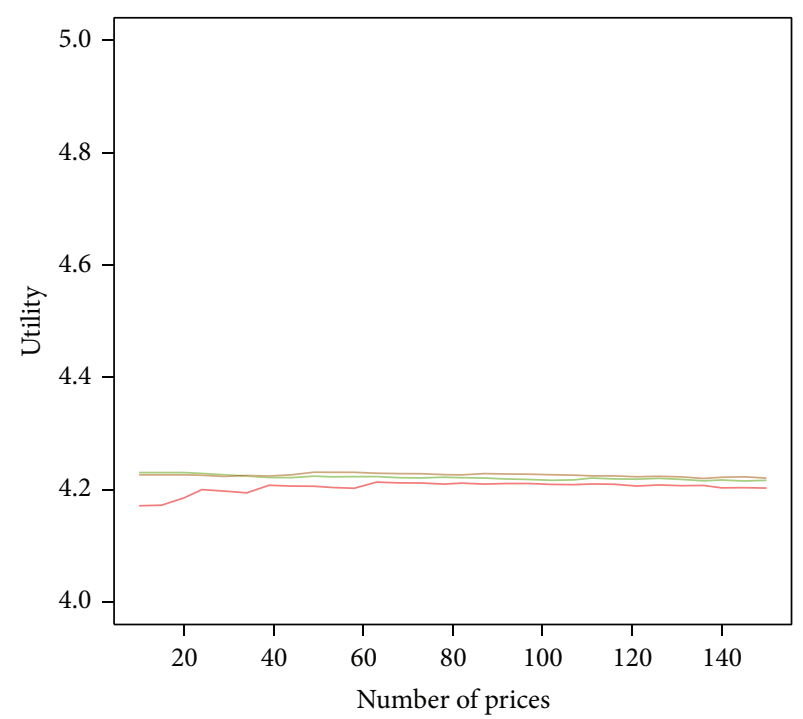

(b) Best utility $U^{*}(N)$ versus $N$

FIGURE 6: Satisficing behavior and induced optimal welfare for a set of distributions with different shapes preserving mean and dispersion (see Table 2).

TABLE 3

\begin{tabular}{lccc}
\hline & $\mu$ & $\sigma$ & $\mathscr{F}$ \\
\hline Green line & 500 & & \\
& 600 & 50 & Normal \\
Red line & 700 & & \\
\hline
\end{tabular}

TABLE 4

\begin{tabular}{lccc}
\hline & $\mu$ & $\sigma$ & $\mathscr{F}$ \\
\hline Green line & & 50 & \\
& 700 & 100 & Normal \\
Red line & & 150 & \\
\hline
\end{tabular}

then the curves $a^{*}(N)$ and correspondence $A(N)$ generated by each distribution coincide. In order to test the reversed implication and, at the same time, to study the response of the consumer behavior to a change in the parameters of the distribution, we compare $a^{*}(N)$ and $A(N)$ for dispersionpreserving distributions with different means and for meanpreserving distributions with different dispersions.

We first take the set of normal distributions $N(\mu, \sigma=$ 50) with means varying in the range $\mu \in\{500,600,700\}$, while all other parameters are kept fixed. Table 3 summarizes the parameter values for this exercise. Figures $7(a)$ and $7(b)$ show the output of the model for this set of distributions. It is apparent that, as the mean increases, both the best satisficer curves $a^{*}(N)$ and the satisficer correspondences $A(N)$ shift downwards. Thus, as the mean price increases, the best satisficer looks over a fewer proportion of price quotes no matter the size of available prices. Also, as it could be expected, the welfare of the best satisficer diminishes uniformly as the mean price increases.
Next we study the changes in the satisficer graphs when a mean preserving normal price distribution becomes more dispersed. The set of normal distributions $N(\mu=700, \sigma)$ with $\sigma \in\{50,100,150\}$ is considered (see Table 4). The results are displayed in Figures 8(a) and 8(b). In contrast with the response with a dispersion-preserving mean shift, now it is observed that both the satisficer correspondences and the best satisficer curve shift upwards when the dispersion increases. Also, when there is a sufficiently large number of price data so that increasing dispersion is noticeable, the utilities of the best satisficer are ordered according to the corresponding dispersions (see Figure 8(b)). Thus, when prices become more dispersed without increasing in mean, the happiest consumer looks over a bigger number of batch prices and in turn her welfare increases.

The normal distribution has been selected above as a benchmark to illustrate the analysis of the model. It can be checked that the qualitative results above also hold for other symmetric distributions. In general, an increase in the mean shifts the best satisficer curve $a^{*}(N)$ downwards whereas an increase in the dispersion has the opposite effect: it shifts the curve $a^{*}(N)$ upwards. Also, the maximizer region and the satisficer zone I (see Figure 5) are affected by changes in the mean and standard deviation: if the price mean increases the maximizer region shrinks and satisficer conducts appear for a lower number of options; whereas if the dispersion increases the satisficer zone I expands and the maximizer behavior remains for a larger number of options.

The numerical study above indicates the following invariance property of the model: the satisficer behaviordescribed by $A(N)$ and $a^{*}(N)$-induced by two different symmetric price distributions changes if and only if the distributions differ either in their means or in their standard deviations-but not in their shapes. 


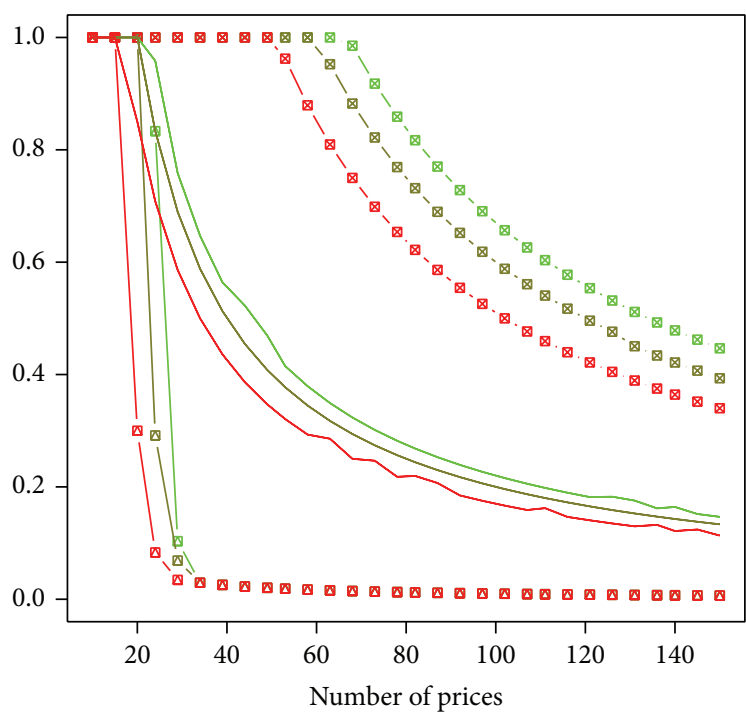

(a) Satisficer correspondences $A(N)$ and best satisficer curves $a^{*}(N)$

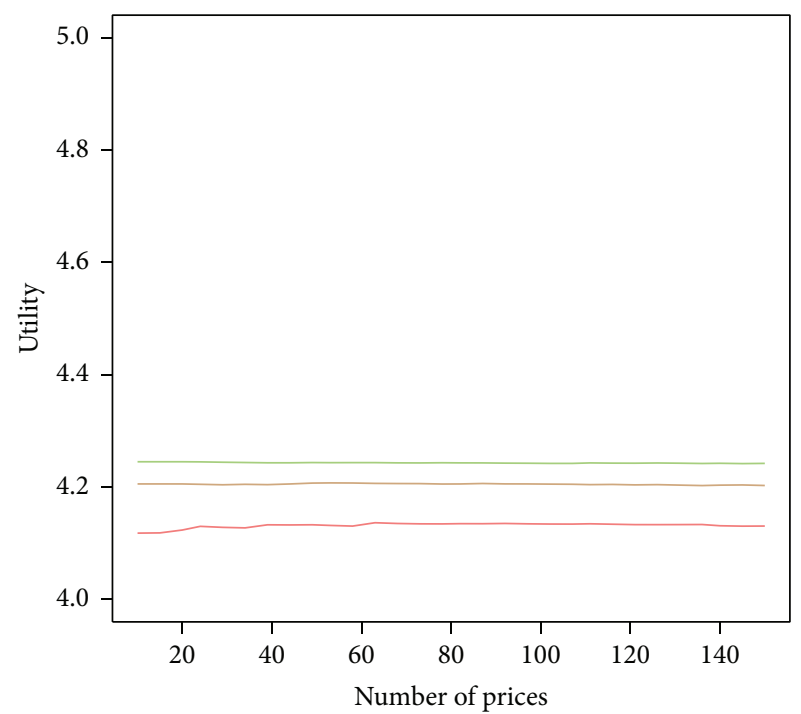

(b) Best utilities $U^{*}(N)$

FIGURE 7: Analysis of satisficing behavior for a dispersion-preserving mean shift under a normal distributions Color of graphs shifts from green to red as $\mu$ increases (see Table 3 ).

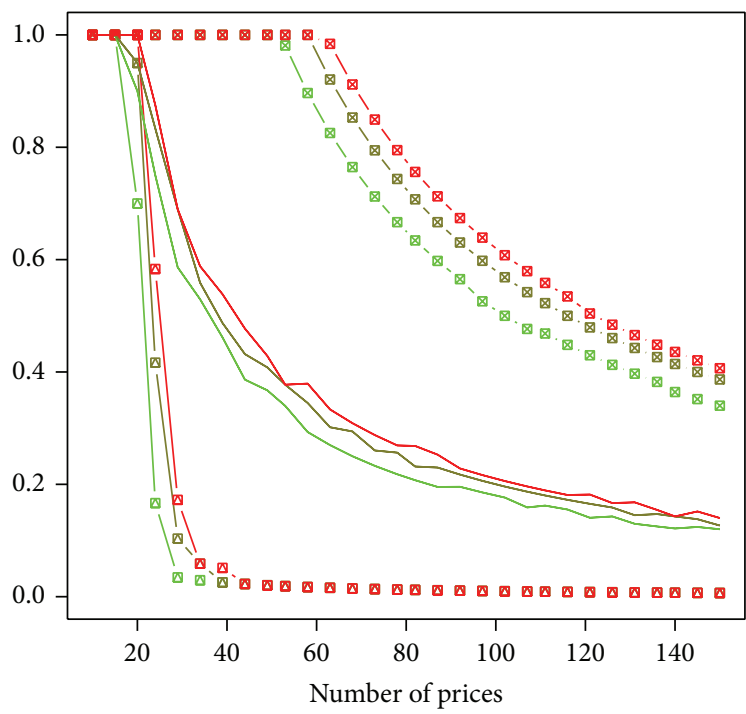

(a) Satisficer correspondences $A(N)$ and best satisficer curves $a^{*}(N)$

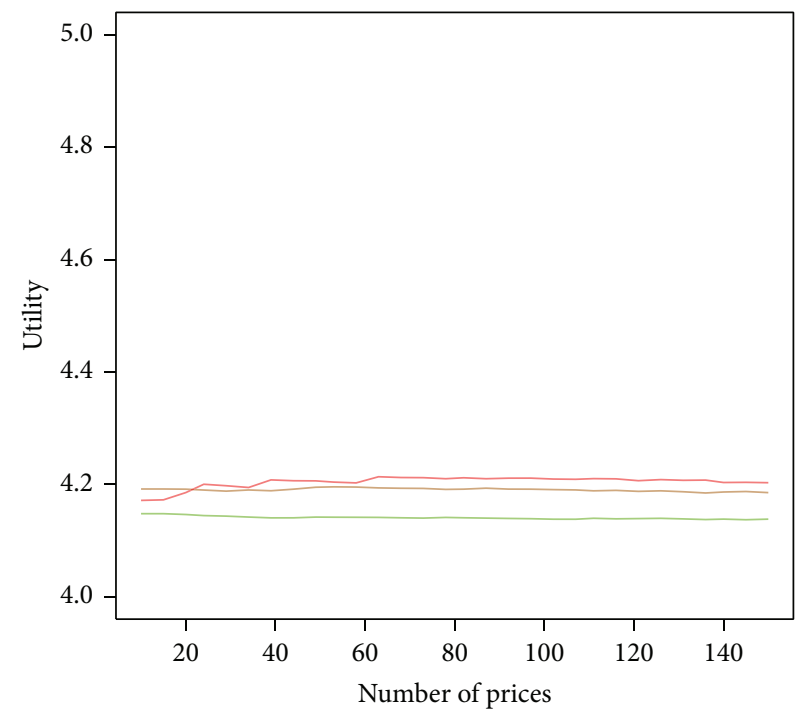

(b) Best utilities $U^{*}(N)$

FIGURE 8: Analysis of satisficing behavior and welfare for mean-preserving dispersion shift under a normal distribution Color of graphs shifts from green to red as $\sigma$ increases (see Table 4 ).

Given that finding a lower price expands the set of affordable time allocations and in turn it might improve welfare, the consumer has an incentive to search for low prices. Since they are to be found at the left tail of the distribution, the symmetry of the distribution may play a crucial role in the invariance property described above. It can thus be suspected that distributions with the same mean and standard deviation but that are asymmetrically dispersed around the mean do not satisfy the invariance property. In order to confirm this claim, we analyze the satisficer behavior for a set of asymmetric distributions with common mean and dispersion. Specifically, we consider split-normal distributions $\mathrm{SN}\left(m, \sigma_{1}, \sigma_{2}\right)$. A split-normal distribution $\mathscr{F}$ is characterized by three moments- $m, \sigma_{1}$, and $\sigma_{2}$ - such that an arbitrary realization of $\mathscr{F}$, say $p$, is distributed $N\left(m, \sigma_{1}\right)$ if $p \leq m$, whereas it is distributed $N\left(m, \sigma_{2}\right)$ if $p>m$. Clearly, the normal (symmetric) distribution is the particular case $\sigma_{1}=\sigma_{2}$, for which $m$ is the mean and the mode. If $\sigma_{1} \neq \sigma_{2}$, then $m$ is mode, while the mean lies to the left (right) of $m$ whenever $\sigma_{1}$ is larger (smaller) than $\sigma_{2}$. Below we 


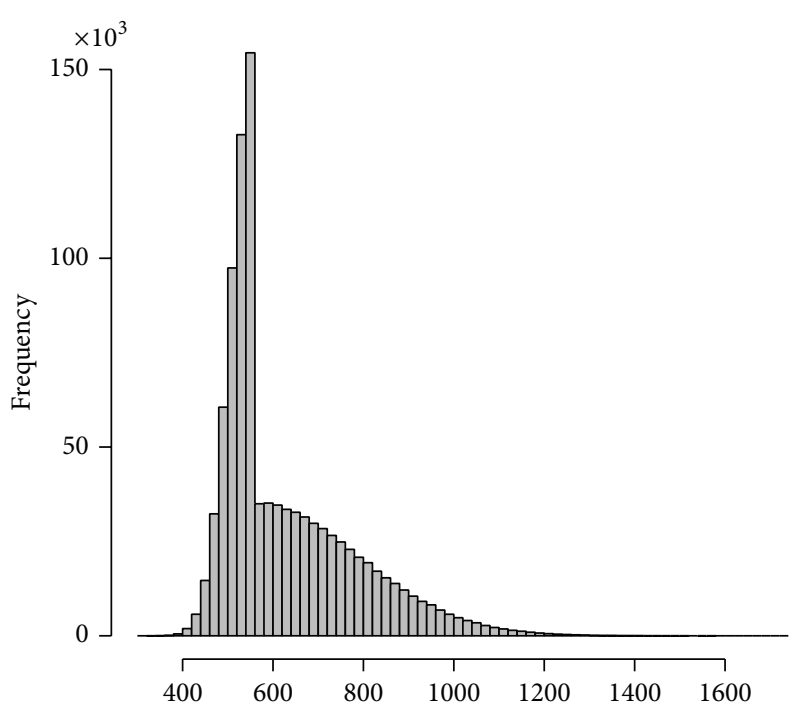

FIgURE 9: Histogram for a split-normal distribution $\mathrm{SN}\left(m=559.82, \sigma_{1}=50, \sigma_{2}=225.68\right)$ with $\mu=700$ and $\sigma=150$ (see Table 5).

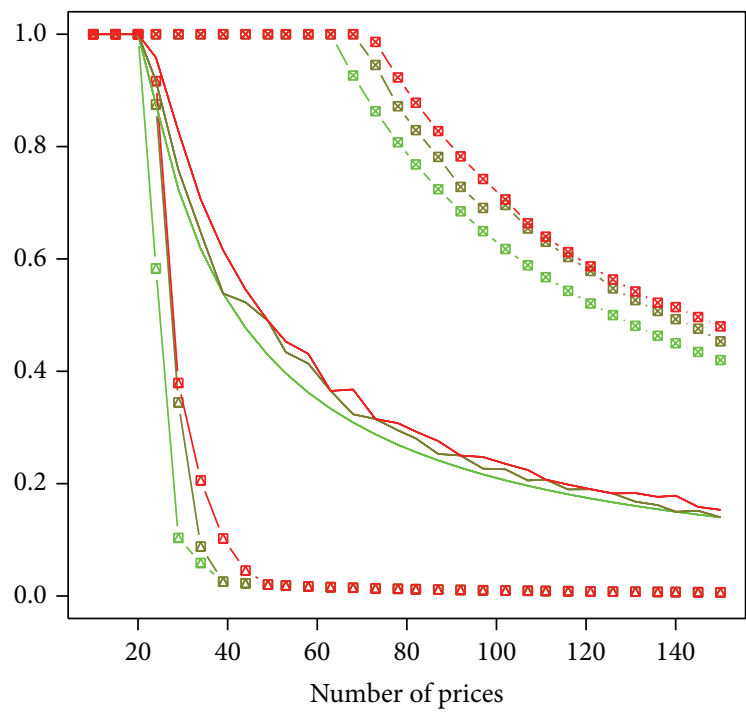

(a) Satisficer correspondences $A(N)$ and best satisficer curves $a^{*}(N)$

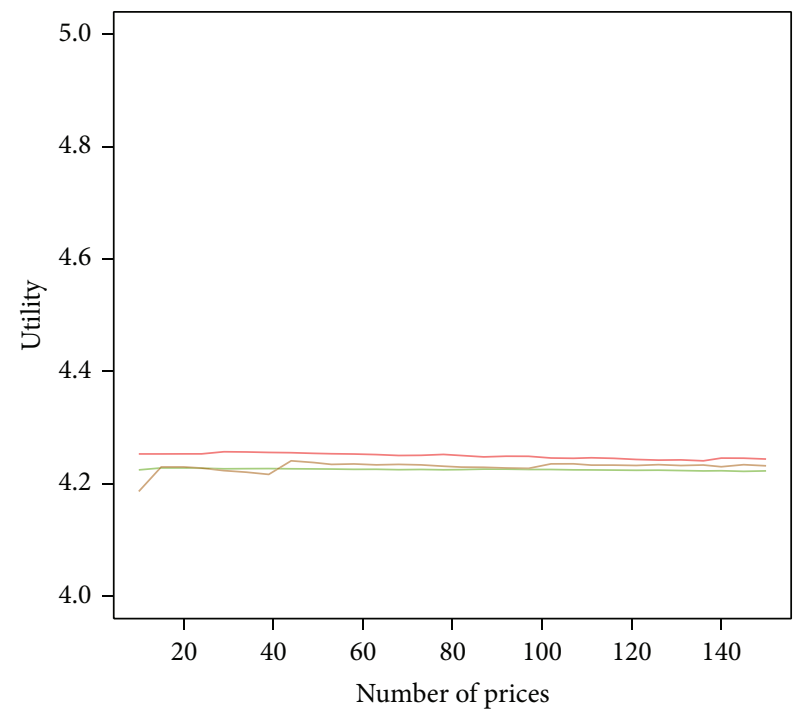

(b) Best utilities $U^{*}(N)$

FIGURE 10: Analysis of satisficing behavior for dispersion and mean preserving split-normal distributions as left dispersion $\sigma_{1}$ increases. Color of graphs shifts from green to red as $\sigma_{1}$ increases (see Table 5).

TABLE 5: The parameters $m$ and $\sigma_{2}$ below are adjusted as $\sigma_{1}$ changes so as to keep $\mu$ and $\sigma$ constant.

\begin{tabular}{lcccc}
\hline & $\mu$ & $\sigma$ & $\mathscr{F}$ & $\sigma_{1}$ \\
\hline Green line & & & & 50 \\
& 700 & \multirow{2}{*}{150} & $\mathrm{SN}\left(m, \sigma_{1}, \sigma_{2}\right)$ & 100 \\
Red line & & & & 150 (Normal) \\
\hline
\end{tabular}

denote the mode by $m$, while we keep $\mu$ and $\sigma$ for mean and standard deviation of $\mathscr{F}$, respectively. A typical asymmetric split-normal histogram can be seen in Figure 9.
The model is solved for a set of three split-normal distributions $\mathrm{SN}\left(m, \sigma_{1}, \sigma_{2}\right)$ with $\sigma_{1} \in\{50,100,150\}$ and with $m$ and $\sigma_{2}$ adjusted in each case to keep the same mean $\mu=700$ and standard deviation $\sigma=150$. The results are displayed in Figures 10(a) and 10(b). Observe that the qualitative changes of the satisficer graphs $A(N)$ and $a^{*}(N)$ as $\sigma_{1}$ increases mimic those of the corresponding graphs in Figures 8(a) and 8(b). That is, as $\sigma_{1}$ increases - in turn the left tail of the distribution becomes thicker-the satisficer graphs shift upwards and the best satisficer improves her welfare. These results show that it is the dispersion on the left tail of the distribution what ultimately modifies the search behavior of satisficers and in 
turn causes the positive effect on her welfare. Furthermore, it confirms that the symmetry of the distribution is required for the invariance property of the model to hold.

\section{Conclusions}

We provide a consumer behavioral model based on rational allocation of time which supports several empirical facts from psychological research. First, the consumer may typically not look over all the product options in the market when searching for a best deal. Second, for a small number of options, consumers who exhaustively look at all product options (maximizers) are better off than consumers who do not consider all options and look over a smaller sample (satisficers). For a large number of options a wide range of satisficers are better off than maximizers. Given a consumer profile and a price distribution for the product options, the model supplies the class of satisficer's strategies that are better off compared with the maximizer's strategy.

Our model analysis confirms a choice overload phenomenon: the higher the number of available options, the lower the fraction of them; a consumer should actually check when seeking her optimal welfare. Remarkably, this is so regardless how prices are distributed in the market. The numerical analysis of the model strongly supports an invariance property with respect to symmetric price distributions, namely, that satisficing conducts depend on the mean and dispersion of prices but not on the shape of the distribution. It is shown that either an increase in the mean or a decrease in the dispersion of prices implies that a relatively less exhaustive search behavior is a better strategy, in terms of welfare, for the consumer. It is also shown that changes in the distribution that preserve mean and standard deviation may alter the satisficer response if the dispersion of the distribution increases only asymmetrically on the left side of the distribution.

The results in this paper support the idea that choice overload phenomena are a behavioral trait of consumers which depends heavily on the number of product options and also on the mean of prices and on the dispersion of low prices. If the distribution of prices in the market is symmetric, its shape plays a limited role in the consumer's satisficing behavior.

\section{Conflict of Interests}

The authors declare that there is no conflict of interests regarding the publication of this paper.

\section{Acknowledgments}

The research in this paper was partially supported by Grant AGL2011-25175 from the Plan Nacional de Investigación Científica of Spain. Raúl G. Sanchis thanks the Complutense Institute for International Studies (ICEI) for its logistic support.

\section{References}

[1] S. S. Iyengar and M. R. Lepper, "When choice is demotivating: can one desire too much of a good thing?" Journal of Personality and Social Psychology, vol. 79, no. 6, pp. 995-1006, 2000.

[2] B. Schwartz, "Self-determination-the tyranny of freedom," American Psychologist, vol. 55, pp. 79-88, 2000.

[3] R. G. Sanchis, J.-M. Rey, and F. Álvarez, "Numerical analysis of a time allocation model accounting for choice overload," International Journal of Computer Mathematics, 2013.

[4] B. Scheibehenne, R. Greifeneder, and P. M. Todd, "Can there ever be too many options? A meta-analytic review of choice overload," Journal of Consumer Research, vol. 37, pp. 409-425, 2010.

[5] A. Caplin, M. Dean, and D. Martin, "Search and satisficing," American Economic Review, vol. 101, no. 7, pp. 2899-2922, 2011.

[6] B. Schwartz, A. Ward, J. Monterosso, S. Lyubomirsky, K. White, and D. R. Lehman, "Maximizing versus satisficing: happiness is a matter of choice," Journal of Personality and Social Psychology, vol. 83, no. 5, pp. 1178-1197, 2002. 


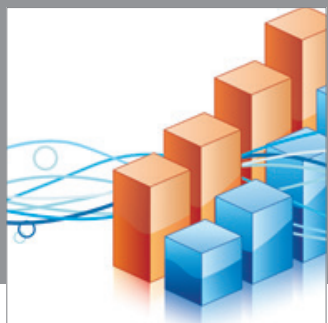

Advances in

Operations Research

mansans

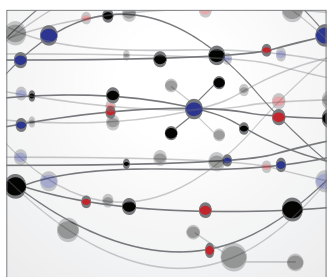

The Scientific World Journal
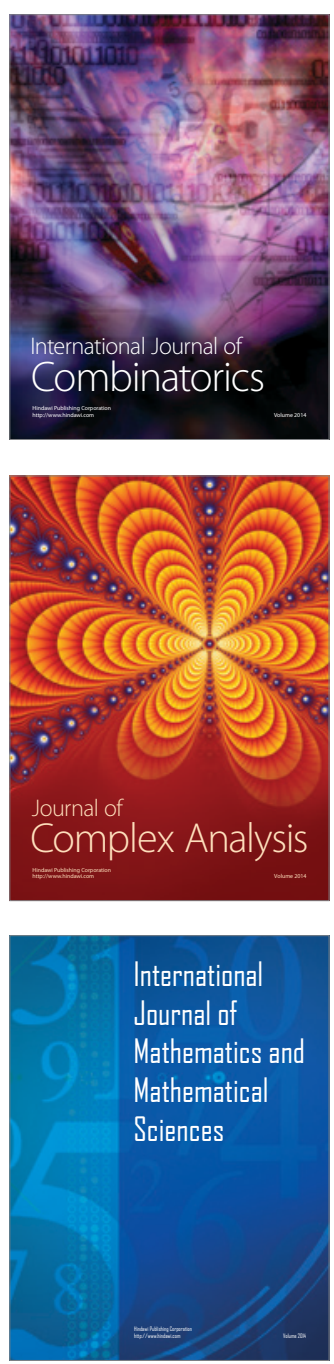
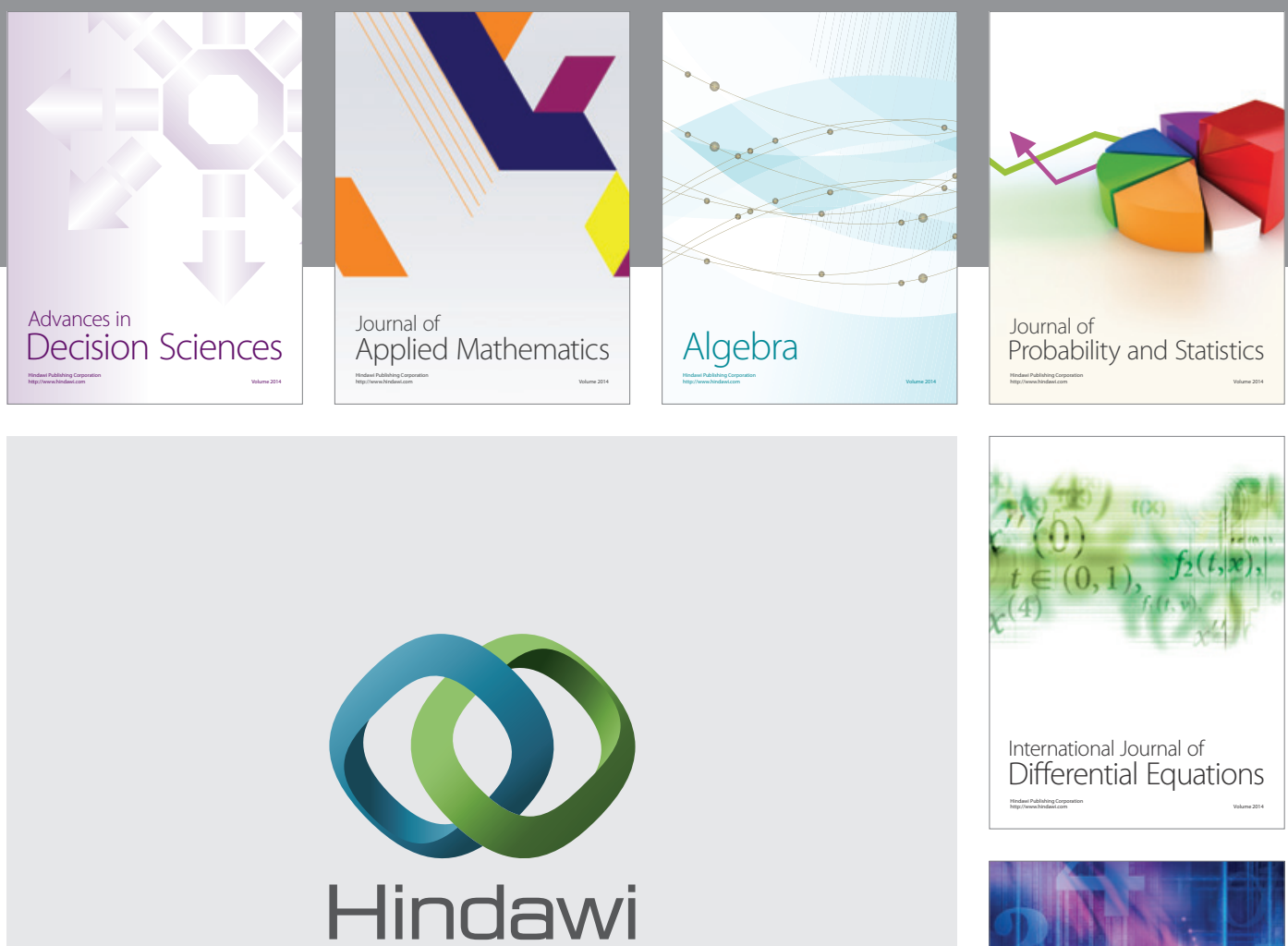

Submit your manuscripts at http://www.hindawi.com
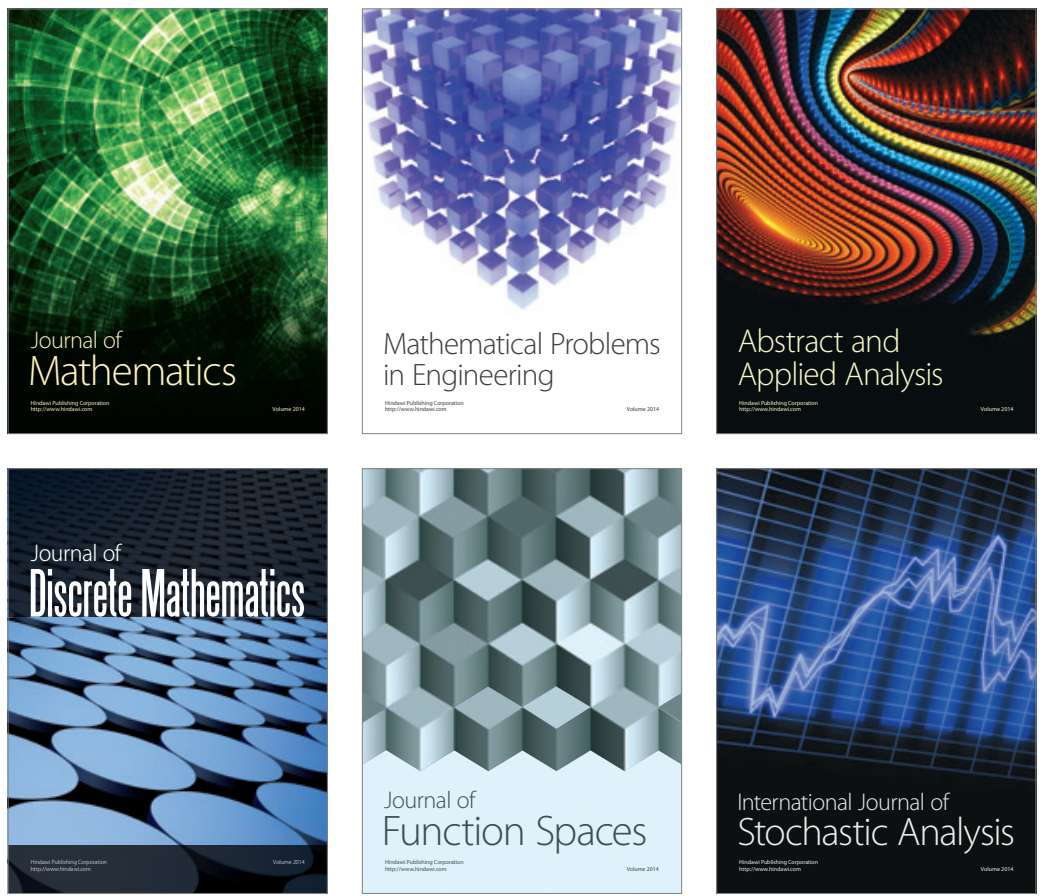

Journal of

Function Spaces

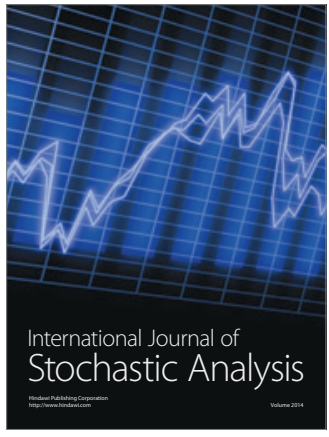

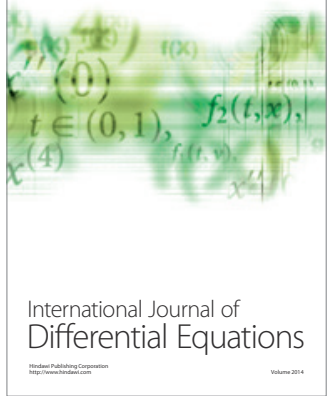
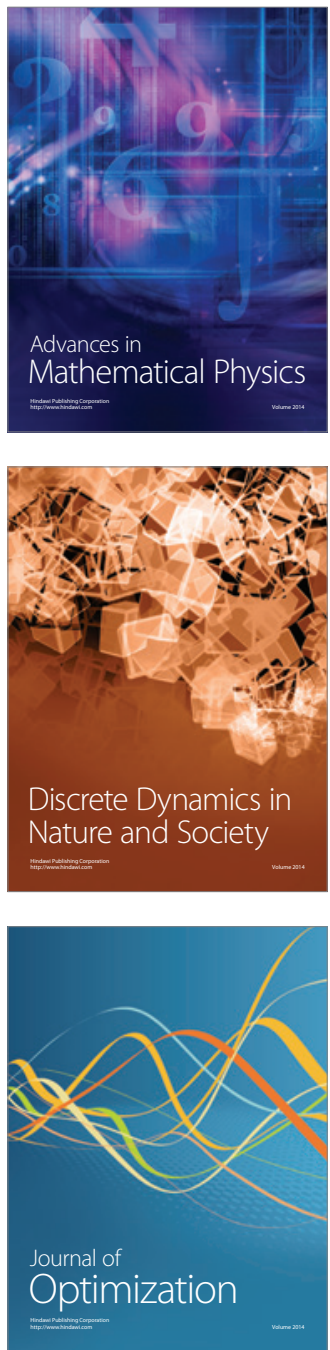